\title{
The phase diagram of neutral quark matter: The effect of neutrino trapping
}

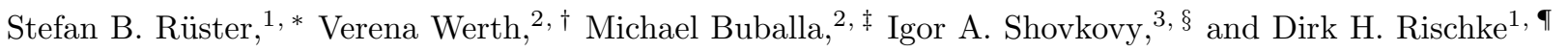 \\ ${ }^{1}$ Institut für Theoretische Physik, J.W. Goethe-Universität, D-60438 Frankfurt am Main, Germany \\ ${ }^{2}$ Institut für Kernphysik, Technische Universität Darmstadt, D-64289 Darmstadt, Germany \\ ${ }^{3}$ Frankfurt Institute for Advanced Studies, J.W. Goethe-Universität, D-60438 Frankfurt am Main, Germany
}

(Dated: November 3, 2006)

\begin{abstract}
We study the effect of neutrino trapping on the phase diagram of dense, locally neutral three-flavor quark matter within the framework of a Nambu-Jona-Lasinio model. In the analysis, dynamically generated quark masses are taken into account self-consistently. The phase diagrams in the plane of temperature and quark chemical potential, as well as in the plane of temperature and leptonnumber chemical potential are presented. We show that neutrino trapping favors two-flavor color superconductivity and disfavors the color-flavor-locked phase at intermediate densities of matter. At the same time, the location of the critical line separating the two-flavor color-superconducting phase and the normal phase of quark matter is little affected by the presence of neutrinos. The implications of these results for the evolution of protoneutron stars are briefly discussed.
\end{abstract}

PACS numbers: 12.39.-x 12.38.Aw 26.60.+c

\section{INTRODUCTION}

Recently, interest in the structure of the phase diagram of neutral dense quark matter has increased considerably [1, 2, 3, 4, 5, 6]. This was driven in part by several key observations regarding the influence of charge neutrality and $\beta$ equilibrium on the competition of various phases in quark matter 7, 8, 9, 10, 11, 12].

In general, dense quark matter is expected to be colorsuperconducting. (For reviews on color superconductivity, see Ref. 13].) At extremely large densities, namely when the quark chemical potential $\mu$ is much larger than the constituent, medium-modified quark masses, the ground state of matter is given by the so-called colorflavor-locked (CFL) phase 14] (for studies of QCD at large densities, see also Ref. 15]). At the highest densities existing in stars, however, the chemical potential is unlikely to be much larger than $500 \mathrm{MeV}$, while the constituent mass of the strange quark is not smaller than the current mass, which is about $100 \mathrm{MeV}$.

In stellar matter, therefore, the heavier strange quark may not be able to participate in diquark Cooper pairing as easily as the light up and down quarks. Then, the pairing of light quarks can lead to the two-flavor colorsuperconducting (2SC) ground state [16, 17]. It should be pointed out, however, that the $2 \mathrm{SC}$ phase is subject to large penalties after imposing the charge neutrality and the $\beta$-equilibrium conditions [7]. Indeed, when the fraction of strange quarks and leptons is small, the electric

\footnotetext{
*Electronic address: ruester@th.physik.uni-frankfurt.de

${ }^{\dagger}$ Electronic address: verena.werth@physik.tu-darmstadt.de

$\ddagger$ Electronic address: michael.buballa@physik.tu-darmstadt.de

$\S$ Electronic address: shovkovy@th.physik.uni-frankfurt.de on leave from Bogolyubov Institute for Theoretical Physics, 03143, Kiev, Ukraine

๑Electronic address: drischke@th.physik.uni-frankfurt.de
}

neutrality requires roughly twice as many down quarks as up quarks. In this case, Cooper pairing of up quarks with down quarks of opposite momenta becomes rather difficult. Then, depending on the details of the interaction, the gapless CFL (gCFL) phase 11, the gapless 2SC (g2SC) phase 10], or even the normal quark (NQ) matter phase may be more favored. Also, there exist other reasonable possibilities 18, 19, 20, 21, 22, 23] which, in view of the known instabilities in the gapless phases [24], are considered to be very promising.

In Ref. [5], we obtained the phase diagram of neutral quark matter, described by the NJL-type model with the parameter set from Ref. 25]. In contrast to previous studies in Refs. 1, 2, 3], the dynamically generated quark masses were treated self-consistently in Ref. [5]. (For earlier studies on color superconductivity, treating quark masses as dynamical quantities, see also Refs. [26, 27].) In the present article we follow the same approach to study the effect of a nonzero neutrino (or, more precisely, lepton-number) chemical potential on the structure of the phase diagram. This is expected to have a potential relevance for the physics of protoneutron stars where neutrinos are trapped during the first few seconds of the stellar evolution.

The effect of neutrino trapping on colorsuperconducting quark matter has been previously discussed in Ref. [8]. It was found that a nonzero neutrino chemical potential favors the $2 \mathrm{SC}$ phase and disfavors the CFL phase. This is not unexpected because the neutrino chemical potential is related to the conserved lepton number in the system and therefore it also favors the presence of (negatively) charged leptons. This helps 2SC-type pairing because electrical neutrality in quark matter can be achieved without inducing a very large mismatch between the Fermi surfaces of up and down quarks. The CFL phase, on the other hand, is electrically and color neutral in the absence of charged leptons when $T=0$ [28]. A nonzero neutrino chemical 
potential can only spoil CFL-type pairing.

In the present paper we extend the analysis of Ref. 8 by performing a more systematic survey of the phase diagram in the space of temperature, quark and leptonnumber chemical potentials. This also includes the possibility of gapless phases, which have not been taken into account in Ref. [8]. While such phases are generally unstable at zero temperature [24], this is not always the case at nonzero temperature [29]. Keeping this in mind, we shall merely localize the "problematic" regions in the phase diagram, where unconventional pairing is unavoidable. We shall refrain, however, from speculating on various possibilities for the true ground state (see, e.g., 18, 19, 20, 21, 22, 23]), since these are still under debate.

In the application to protoneutron stars, it is of interest to cover a range of parameters that could provide a total lepton fraction in quark matter of up to about 0.4. This is the value of the lepton-to-baryon charge ratio in iron cores of progenitor stars. Because of the conservation of both lepton and baryon charges, this value is also close to the lepton fraction in protoneutron stars at early times, when the leptons did not have a chance to diffuse through dense matter and escape from the star [30]. The densities in the central regions of protoneutron stars can reach values of up to about 10 times larger than the nuclear saturation density $\rho_{0} \approx 0.15 \mathrm{fm}^{-3}[30$. In the model studied in the present paper, almost the whole range of possibilities shall be covered by restricting the quark chemical potential to $\mu \lesssim 500 \mathrm{MeV}$ and the neutrino chemical potential to $\mu_{\nu_{e}} \lesssim 400 \mathrm{MeV}$.

This paper is organized as follows. In Sec. III we briefly introduce the model. The general effects of neutrino trapping on two- and three-flavor quark matter are discussed in Sec. III] The results for the phase diagram of quark matter with neutrino trapping are presented in Sec. IV] In Sec. $\nabla$ we summarize our results and discuss their implications for the evolution of protoneutron stars.

\section{MODEL}

We consider a system of up, down, and strange quarks, in weak equilibrium with charged leptons and the corresponding neutrinos. We assume that the lepton sector of the model is given by an ideal gas of massive electrons $\left(m_{e} \approx 0.511 \mathrm{MeV}\right)$ and muons $\left(m_{\mu} \approx 105.66 \mathrm{MeV}\right)$, as well as massless electron and muon neutrinos. We do not take into account the $\tau$ lepton, which is too heavy to play any role in dense matter. Also, we neglect the possibility of neutrino mixing, and therefore we do not take into account the $\tau$ neutrino either.

In the quark sector, we use the same three-flavor Nambu-Jona-Lasinio model as in Ref. [5]. The La- grangian density reads

$$
\begin{aligned}
\mathcal{L} & =\bar{\psi}(i \not \partial-\hat{m}) \psi+G_{S} \sum_{a=0}^{8}\left[\left(\bar{\psi} \lambda_{a} \psi\right)^{2}+\left(\bar{\psi} i \gamma_{5} \lambda_{a} \psi\right)^{2}\right] \\
& +G_{D} \sum_{\gamma, c}\left[\bar{\psi}_{\alpha}^{a} i \gamma_{5} \epsilon^{\alpha \beta \gamma} \epsilon_{a b c}\left(\psi_{C}\right)_{\beta}^{b}\right]\left[\left(\bar{\psi}_{C}\right)_{\rho}^{r} i \gamma_{5} \epsilon^{\rho \sigma \gamma} \epsilon_{r s c} \psi_{\sigma}^{s}\right] \\
& -K\left\{\operatorname{det}_{f}\left[\bar{\psi}\left(1+\gamma_{5}\right) \psi\right]+\operatorname{det}_{f}\left[\bar{\psi}\left(1-\gamma_{5}\right) \psi\right]\right\},
\end{aligned}
$$

where the quark spinor field $\psi_{\alpha}^{a}$ carries color $(a=r, g, b)$ and flavor $(\alpha=u, d, s)$ indices. $\hat{m}=\operatorname{diag}_{f}\left(m_{u}, m_{d}, m_{s}\right)$ is the matrix of quark current masses; $\lambda_{a}(a=1, \ldots, 8)$ are the Gell-Mann matrices in flavor space, and $\lambda_{0} \equiv$ $\sqrt{2 / 3} \mathbb{1}_{f}$. The charge conjugate spinor is defined as $\psi_{C}=$ $C \bar{\psi}^{T}$ and $\bar{\psi}_{C}=\psi^{T} C$, where $\bar{\psi}=\psi^{\dagger} \gamma^{0}$ is the Dirac conjugate spinor and $C=i \gamma^{2} \gamma^{0}$ is the charge conjugation matrix.

In the model at hand, there are six mutually commuting conserved charge densities. They split naturally into the following three classes.

(i) Electric charge. This is related to the $U(1)$ symmetry of electromagnetism. The corresponding charge density is given by

$$
n_{Q}=\left\langle\psi^{\dagger} Q \psi\right\rangle-n_{e}-n_{\mu},
$$

where $Q=\operatorname{diag}_{f}\left(\frac{2}{3},-\frac{1}{3},-\frac{1}{3}\right)$ is the electric charge matrix of the quarks, and $n_{e}$ and $n_{\mu}$ denote the number densities of electrons and muons, respectively.

(ii) Two lepton charges. As long as the neutrinos are trapped and their oscillations are neglected, the lepton family numbers are conserved. The corresponding densities read

$$
n_{L_{e}}=n_{e}+n_{\nu_{e}}, \quad n_{L_{\mu}}=n_{\mu}+n_{\nu_{\mu}} .
$$

(iii) Baryon number and two color charges. The $S U(3)$ color symmetry and $U(1)$ baryon number symmetry imply the conservation of three independent charge densities in the quark sector,

$n=\left\langle\psi^{\dagger} \psi\right\rangle, \quad n_{3}=\left\langle\psi^{\dagger} T_{3} \psi\right\rangle, \quad n_{8}=\left\langle\psi^{\dagger} T_{8} \psi\right\rangle$,

where $T_{3}=\operatorname{diag}_{c}\left(\frac{1}{2},-\frac{1}{2}, 0\right)$ and $\sqrt{3} T_{8}=$ $\operatorname{diag}_{c}\left(\frac{1}{2}, \frac{1}{2},-1\right)$ are the matrices associated with the two mutually commuting color charges of the $S U(3)_{c}$ gauge group. Note that $n$ is the quark number density that is related to the baryon number density as follows: $n_{B}=\frac{1}{3} n$. An alternative choice of the three conserved charges is given by the number densities of red, green, and blue quarks, i.e., $n_{r}=n_{B}+n_{3}+n_{8} / \sqrt{3}, n_{g}=n_{B}-n_{3}+n_{8} / \sqrt{3}$, and $n_{b}=n_{B}-2 n_{8} / \sqrt{3}$. 
The six conserved charge densities defined above are related to the six chemical potentials of the model. These are the quark chemical potential $\mu=\mu_{B} / 3$, the two color chemical potentials $\mu_{3}$ and $\mu_{8}$, the electric charge chemical potential $\mu_{Q}$, and the two lepton-number chemical potentials $\mu_{L_{e}}$ and $\mu_{L_{\mu}}$.

In chemical equilibrium, the chemical potentials of all individual quark and lepton species can be expressed in terms of these six chemical potentials according to their content of conserved charges. For the quarks, which carry quark number, color, and electric charge, this is related to the diagonal elements of the following matrix:

$\mu_{a b}^{\alpha \beta}=\left(\mu \delta^{\alpha \beta}+\mu_{Q} Q^{\alpha \beta}\right) \delta_{a b}+\left[\mu_{3}\left(T_{3}\right)_{a b}+\mu_{8}\left(T_{8}\right)_{a b}\right] \delta^{\alpha \beta}$,

i.e., the chemical potential of a quark with flavor $\alpha$ and color $a$ is given by $\mu_{a}^{\alpha} \equiv \mu_{a a}^{\alpha \alpha}$. The neutrinos, on the other hand, carry lepton number only. Then,

$$
\mu_{\nu_{e}}=\mu_{L_{e}}, \quad \mu_{\nu_{\mu}}=\mu_{L_{\mu}} .
$$

Finally, electrons and muons carry both, lepton number and electric charge, hence

$$
\mu_{e}=\mu_{L_{e}}-\mu_{Q}, \quad \mu_{\mu}=\mu_{L_{\mu}}-\mu_{Q} .
$$

As in Ref. [5], the quark part of our model is treated in the mean-field (Hartree) approximation, allowing for the presence of both quark-antiquark condensates and scalar diquark condensates. In the model, the constituent quark masses are defined by the following relation:

$$
M_{\alpha}=m_{\alpha}-4 G_{S} \sigma_{\alpha}+2 K \sigma_{\beta} \sigma_{\gamma},
$$

where $\sigma_{\alpha}=\left\langle\bar{\psi}_{\alpha}^{a} \psi_{\alpha}^{a}\right\rangle$ (no sum over $\alpha$ ) are the quarkantiquark condensates, and the set of indices $(\alpha, \beta, \gamma)$ is a permutation of $(u, d, s)$.

Diquark pairing in the model is described by three gap parameters

$$
\Delta_{c}=2 G_{D}\left\langle\left(\bar{\psi}_{C}\right)_{\alpha}^{a} i \gamma_{5} \epsilon^{\alpha \beta c} \epsilon_{a b c} \psi_{\beta}^{b}\right\rangle
$$

(no sum over $c$ ). By definition, the parameters $\Delta_{1}, \Delta_{2}$, and $\Delta_{3}$ correspond to the down-strange, up-strange, and up-down diquark condensates, respectively.

By following the same steps in the derivation as in Ref. [5] and including the ideal-gas contributions for the leptons, we arrive at the following expression for the pressure:

$$
\begin{aligned}
p & =\frac{1}{2 \pi^{2}} \sum_{i=1}^{18} \int_{0}^{\Lambda} \mathrm{d} k k^{2}\left[\left|\epsilon_{i}\right|+2 T \ln \left(1+\mathrm{e}^{-\frac{\left|\epsilon_{i}\right|}{T}}\right)\right] \\
& +4 K \sigma_{u} \sigma_{d} \sigma_{s}-\frac{1}{4 G_{D}} \sum_{c=1}^{3}\left|\Delta_{c}\right|^{2}-2 G_{S} \sum_{\alpha=1}^{3} \sigma_{\alpha}^{2} \\
& +\sum_{l=e, \mu}\left[\frac{T}{\pi^{2}} \sum_{\epsilon= \pm} \int_{0}^{\infty} \mathrm{d} k k^{2} \ln \left(1+\mathrm{e}^{-\frac{E_{l}-\epsilon \mu_{l}}{T}}\right)\right. \\
& \left.+\frac{1}{24 \pi^{2}}\left(\mu_{\nu_{l}}^{4}+2 \pi^{2} \mu_{\nu_{l}}^{2} T^{2}+\frac{7}{15} \pi^{4} T^{4}\right)\right]
\end{aligned}
$$

where $\epsilon_{i}$ are eighteen independent positive-energy eigenvalues, see Ref. [5] for details. The pressure in Eq. (10) has a physical meaning only at stationary points with respect to variations of the chiral and colorsuperconducting condensates. Thus, $\sigma_{\alpha}$ and $\Delta_{c}$ must satisfy the following set of six gap equations:

$$
\begin{aligned}
\frac{\partial p}{\partial \sigma_{\alpha}} & =0, \\
\frac{\partial p}{\partial \Delta_{c}} & =0 .
\end{aligned}
$$

To enforce the conditions of local charge neutrality in dense matter, one also requires that the following three equations are satisfied:

$$
\begin{aligned}
n_{Q} & \equiv \frac{\partial p}{\partial \mu_{Q}}=0, \\
n_{3} & \equiv \frac{\partial p}{\partial \mu_{3}}=0, \\
n_{8} & \equiv \frac{\partial p}{\partial \mu_{8}}=0 .
\end{aligned}
$$

We should note that, in general, the restriction to the color charges $n_{3}$ and $n_{8}$ is not sufficient, but one has to ensure that the densities $n_{a}=\left\langle\psi^{\dagger} T_{a} \psi\right\rangle$ vanish for all generators $T_{a}$ of $S U(3)_{c}$, i.e., $a=1, \ldots, 8$ 31]. However, for all phases we are considering here, we have checked explicitly that the condition $n_{a}=0$ is automatically satisfied for $a \neq 3,8$. Thus, we are left with Eqs. (12). By solving these, we determine the values of the three corresponding chemical potentials $\mu_{Q}, \mu_{3}$, and $\mu_{8}$ for a given set of the other chemical potentials, $\mu, \mu_{L_{e}}, \mu_{L_{\mu}}$, and for a given temperature $T$. In general, therefore, the phase diagram of dense quark matter with neutrino trapping should span a four-dimensional parameter space.

Note that instead of using the chemical potentials, $\mu$, $\mu_{L_{e}}$, and $\mu_{L_{\mu}}$ as free parameters in the study of the phase diagram, one may also try to utilize the quark number density and the two lepton fractions,

$$
\begin{aligned}
n & \equiv \frac{\partial p}{\partial \mu}, \\
Y_{L_{e}} & \equiv 3 \frac{n_{L_{e}}}{n}, \\
Y_{L_{\mu}} & \equiv 3 \frac{n_{L_{\mu}}}{n},
\end{aligned}
$$

where the two lepton densities $n_{L_{i}}$ are defined by

$$
n_{L_{e}} \equiv \frac{\partial p}{\partial \mu_{L_{e}}}, \quad n_{L_{\mu}} \equiv \frac{\partial p}{\partial \mu_{L_{\mu}}} .
$$

In some cases, the choice of $n, Y_{L_{e}}$ and $Y_{L_{\mu}}$ as free parameters is indeed very useful. For instance, this is helpful in order to determine the initial state of matter inside the protoneutron star at very early times, when the lepton fractions are approximately the same as in the progenitor star (i.e., $Y_{L_{e}} \approx 0.4$ and $Y_{L_{\mu}}=0$ ). The problem is, however, that such an approach becomes ambiguous in the 
vicinity of first-order phase transitions, where the baryon number density as well as the lepton fractions are in general discontinuous. For this reason, it is more appropriate to study the phase structure of (dense) QCD at given fixed values of the chemical potentials $\mu, \mu_{L_{e}}$, and $\mu_{L_{\mu}}$. Unlike densities, the chemical potentials change continuously when the system crosses a boundary of a first-order phase transition. (Here it should be noted that, because of the long-range Coulomb interaction enforcing the constraints $n_{Q}=0, n_{3}=0$ and $n_{8}=0$, the chemical potentials $\mu_{Q}, \mu_{3}$, and $\mu_{8}$ may change almost discontinuously at a boundary of a first-order phase transition.)

\section{SIMPLIFIED CONSIDERATIONS}

As mentioned in the Introduction, neutrino trapping favors the 2SC phase and strongly disfavors the CFL phase [8]. This is a consequence of the modified $\beta$ equilibrium condition in the system. In this section, we would like to emphasize that this is a model-independent effect. In order to understand the physics behind it, it is instructive to start our consideration from a very simple toy model. Later, many of its qualitative features will be also observed in our self-consistent numerical analysis of the NJL model.

Let us first assume that strange quarks are very heavy and consider a gas of non-interacting massless up and down quarks in the normal phase at $T=0$. As required by $\beta$ equilibrium, electrons and electron neutrinos are also present in the system. (Note that in this section we neglect muons and muon neutrinos for simplicity.)

In the absence of Cooper pairing, the densities of quarks and leptons are given by

$$
n_{u, d}=\frac{\mu_{u, d}^{3}}{\pi^{2}}, \quad n_{e}=\frac{\mu_{e}^{3}}{3 \pi^{2}}, \quad n_{\nu_{e}}=\frac{\mu_{\nu_{e}}^{3}}{6 \pi^{2}} .
$$

Expressing the chemical potentials through $\mu, \mu_{Q}$ and $\mu_{L_{e}}$, and imposing electric charge neutrality, one arrives at the following relation:

$$
2\left(1+\frac{2}{3} y\right)^{3}-\left(1-\frac{1}{3} y\right)^{3}-(x-y)^{3}=0,
$$

where we have introduced the chemical potential ratios $x=\mu_{L_{e}} / \mu$ and $y=\mu_{Q} / \mu$. The above cubic equation can be solved for $y$ (electric chemical potential) at any given $x$ (lepton-number chemical potential). The result can be used to calculate the ratio of quark chemical potentials, $\mu_{d} / \mu_{u}=(3-y) /(3+2 y)$.

The ratio $\mu_{d} / \mu_{u}$ as a function of $\mu_{L_{e}} / \mu$ is shown in Fig. 11 At vanishing $\mu_{L_{e}}$, one finds $y \approx-0.219$ and, thus, $\mu_{d} / \mu_{u} \approx 1.256$ (note that this value is very close to $2^{1 / 3} \approx$ 1.260). This result corresponds to the following ratios of the number densities in the system: $n_{u} / n_{d} \approx 0.504$ and $n_{e} / n_{d} \approx 0.003$, reflecting that the density of electrons is tiny and the charge of the up quarks has to be balanced by approximately twice as many down quarks.
At $\mu_{L_{e}}=\mu$, on the other hand, the real solution to Eq. (16) is $y=0$, i.e., the up and down Fermi momenta become equal. This can be seen most easily if one inverts the problem and solves Eq. (16) for $x$ at given $y$. When $y=0$ one finds $x=1$, meaning that $\mu_{d}=\mu_{u}$ and, in turn, suggesting that pairing between up and down quarks is unobstructed at $\mu_{L_{e}}=\mu$. This is in contrast to the case of vanishing $\mu_{L_{e}}$, when the two Fermi surfaces are split by about $25 \%$, and pairing is difficult.

It is appropriate to mention that many features of the above considerations would not change much even when Cooper pairing is taken into account. The reason is that the corresponding corrections to the quark densities are parametrically suppressed by a factor of order $(\Delta / \mu)^{2}$.

In order to estimate the magnitude of the effect in the case of dense matter in protoneutron stars, we indicate several typical values of the lepton fractions $Y_{L_{e}}$ in Fig. 1] As mentioned earlier, $Y_{L_{e}}$ is expected to be of order 0.4 right after the collapse of the iron core of a progenitor star. According to Fig. [1] this corresponds to $\mu_{d} / \mu_{u} \approx 1.1$, i.e., while the splitting between the up and down Fermi surfaces does not disappear completely, it gets reduced considerably compared to its value in the absence of trapped neutrinos. This reduction substantially facilitates the cross-flavor pairing of up and down quarks. The effect is gradually washed out during about a dozen of seconds of the deleptonization period when the value of $Y_{L_{e}}$ decreases to zero.

The toy model is easily modified to the opposite extreme of three massless quark flavors. Basically, this corresponds to replacing Eq. (16) by

$$
2\left(1+\frac{2}{3} y\right)^{3}-2\left(1-\frac{1}{3} y\right)^{3}-(x-y)^{3}=0 .
$$

In the absence of neutrino trapping, $x=0$, the only real solution to this equation is $y=0$, indicating that the chemical potentials (which also coincide with the Fermi momenta) of up, down, and strange quarks are equal. This reflects the fact that the system with equal densities of up, down, and strange quarks is neutral by itself, without electrons. With increasing $x \propto \mu_{L_{e}}$, the solution requires a nonzero $y \propto \mu_{Q}$, suggesting that up-down and up-strange pairing becomes more difficult. To see this more clearly, we can go one step further in the analysis of the toy model.

Let us assume that the quarks are paired in a regular, i.e., fully gapped, CFL phase at $T=0$. Then, as shown in Ref. [28], the quark part of the matter is automatically electrically neutral. Hence, if we want to keep the whole system electrically and color neutral, there must be no electrons. Obviously, this is easily realized without trapped neutrinos by setting $\mu_{Q}$ equal to zero. At non-vanishing $\mu_{L_{e}}$ the situation is more complicated. The quark part is still neutral by itself and therefore no electrons are admitted. Hence, the electron chemical potential $\mu_{e}=\mu_{L_{e}}-\mu_{Q}$ must vanish, and consequently $\mu_{Q}$ should be nonzero and equal to $\mu_{L_{e}}$. It is natural to ask what should be the values of the color chemical potentials 
$\mu_{3}$ and $\mu_{8}$ in the CFL phase when $\mu_{L_{e}} \neq 0$.

In order to analyze the stress on the CFL phase due to nonzero $\mu_{L_{e}}$, we follow the same approach as in Refs. 7, 11]. In this analytical consideration, we also account for the effect of the strange quark mass simply by shifting the strange quark chemical potential by $-M_{s}^{2} /(2 \mu)$. In our notation, pairing of CFL-type requires the following "common" values of the Fermi momenta of paired quarks:

$$
\begin{aligned}
p_{F,(r u, g d, b s)}^{\text {common }} & =\mu-\frac{M_{s}^{2}}{6 \mu}, \\
p_{F,(r d, g u)}^{\text {common }} & =\mu+\frac{\mu_{Q}}{6}+\frac{\mu_{8}}{2 \sqrt{3}}, \\
p_{F,(r s, b u)}^{\text {common }} & =\mu+\frac{\mu_{Q}}{6}+\frac{\mu_{3}}{4}-\frac{\mu_{8}}{4 \sqrt{3}}-\frac{M_{s}^{2}}{4 \mu}, \\
p_{F,(g s, b d)}^{\text {common }} & =\mu-\frac{\mu_{Q}}{3}-\frac{\mu_{3}}{4}-\frac{\mu_{8}}{4 \sqrt{3}}-\frac{M_{s}^{2}}{4 \mu} .
\end{aligned}
$$

These are used to calculate the pressure in the toy model,

$$
\begin{aligned}
p^{(\text {toy })}= & \frac{1}{\pi^{2}} \sum_{a=1}^{3} \sum_{\alpha=1}^{3} \int_{0}^{p_{F, a \alpha}^{\text {common }}}\left(\mu_{a}^{\alpha}-p\right) p^{2} d p \\
& +3 \frac{\mu^{2} \Delta^{2}}{\pi^{2}}+\frac{\left(\mu_{L_{e}}-\mu_{Q}\right)^{4}}{12 \pi^{2}}+\frac{\mu_{L_{e}}^{4}}{24 \pi^{2}} .
\end{aligned}
$$

By making use of this expression, one easily derives the neutrality conditions as in Eq. (12). In order to solve them, it is useful to note that

$$
n_{Q}-n_{3}-\frac{1}{\sqrt{3}} n_{8}=\frac{\left(\mu_{Q}-\mu_{L_{e}}\right)^{3}}{3 \pi^{2}} .
$$

Thus, it becomes obvious that charge neutrality requires $\mu_{Q}=\mu_{L_{e}}$. The other useful observation is that the expression for $n_{3}$ is proportional to $\mu_{3}+\mu_{Q}$. So, it is vanishing if (and only if) $\mu_{3}=-\mu_{Q}$, which means that $\mu_{3}=-\mu_{L_{e}}$. Finally, one can check that the third neutrality condition $n_{8}=0$ requires

$$
\mu_{8}=-\frac{\mu_{L_{e}}}{\sqrt{3}}-\frac{M_{s}^{2}}{\sqrt{3} \mu} .
$$

The results for the charge chemical potentials $\mu_{Q}, \mu_{3}$, and $\mu_{8}$ imply the following magnitude of stress on pairing in the CFL phase:

$$
\begin{aligned}
& \delta \mu_{(r d, g u)}=\frac{\mu_{g}^{u}-\mu_{r}^{d}}{2}=\mu_{L_{e}}, \\
& \delta \mu_{(r s, b u)}=\frac{\mu_{b}^{u}-\mu_{r}^{s}}{2}=\mu_{L_{e}}+\frac{M_{s}^{2}}{2 \mu}, \\
& \delta \mu_{(g s, b d)}=\frac{\mu_{b}^{d}-\mu_{g}^{s}}{2}=\frac{M_{s}^{2}}{2 \mu} .
\end{aligned}
$$

Note that there is no mismatch between the values of the chemical potentials of the other three quarks, $\mu_{r}^{u}=$ $\mu_{g}^{d}=\mu_{b}^{s}=\mu-M_{s}^{2} /(6 \mu)$.
From Eq. (22) we see that the largest mismatch occurs in the $(r s, b u)$ pair (for positive $\left.\mu_{L_{e}}\right)$. The CFL phase can withstand the stress only if the value of $\delta \mu_{(r s, b u)}$ is less than $\Delta_{2}$. A larger mismatch should drive a transition to a gapless phase exactly as in Refs. 10, 11]. Thus, the critical value of the lepton-number chemical potential is

$$
\mu_{L_{e}}^{(\mathrm{cr})} \approx \Delta_{2}-\frac{M_{s}^{2}}{2 \mu} .
$$

When $\mu_{L_{e}}>\mu_{L_{e}}^{(\mathrm{cr})}$, the CFL phase turns into the gCFL' phase, which is a variant of the gCFL phase 11]. By definition, the gapless mode with a linear dispersion relation in the gCFL' phase is $r s-b u$ instead of $g s-b d$ as in the standard gCFL phase. (Let us remind that the mode $a \alpha-$ $b \beta$ is defined by its dispersion relation which interpolates between the dispersion relations of hole-type excitations of $a \alpha$-quark at small momenta, $k \ll \mu_{a}^{\alpha}$, and particle-type excitations of $b \beta$-quark at large momenta, $k \gg \mu_{b}^{\beta}$.)

In order to see what this means for the physics of protoneutron stars, we should again try to relate the value of $\mu_{L_{e}}$ to the lepton fraction. As we have seen, there are no electrons in the (regular) CFL phase at $T=0$. Therefore the entire lepton number is carried by neutrinos. For the baryon density we may neglect the pairing effects to first approximation and employ the ideal-gas relations. This yields

$$
Y_{L_{e}} \approx \frac{1}{6}\left(\frac{\mu_{L_{e}}}{\mu}\right)^{3}
$$

Inserting typical numbers, $\mu=500 \mathrm{MeV}$ and $\mu_{L_{e}} \lesssim \Delta \approx$ $50-100 \mathrm{MeV}$, one finds $Y_{L_{e}} \lesssim 10^{-4}-10^{-3}$. Thus, there is practically no chance to find a sizable amount of leptons in the CFL phase. The constraint gets relaxed slightly at nonzero temperatures and/or in the gCFL phase, but the lepton fraction remains rather small even then (our numerical results indicate that, in general, $Y_{L_{e}} \lesssim 0.05$ in the CFL phase).

\section{RESULTS}

The simple toy-model considerations in the previous section give a qualitative understanding of the effect of neutrino trapping on the mismatch of the quark Fermi momenta and, thus, on the pairing properties of twoand three-flavor quark matter. Now, we turn to a more detailed numerical analysis of the phase diagram in the NJL model defined in Sec. III

In the numerical calculations, we use the following set of model parameters [25]:

$$
\begin{aligned}
\Lambda & =602.3 \mathrm{MeV}, \\
G_{S} \Lambda^{2} & =1.835 \\
K \Lambda^{5} & =12.36 \\
m_{s} & =140.7 \mathrm{MeV}, \\
m_{u, d} & =5.5 \mathrm{MeV} .
\end{aligned}
$$


The parameters are chosen to reproduce several key observables of vacuum QCD 25]. In this paper, our choice for the diquark coupling is $G_{D}=\frac{3}{4} G_{S}$, corresponding to what is called "intermediate coupling" in Ref. 5].

In order to obtain the phase diagram, one has to determine the ground state of matter for each given set of the parameters. As discussed in Sec. II in the case of locally neutral matter with trapped neutrinos, there are four parameters that should be specified: the temperature $T$, the quark chemical potential $\mu$ as well as the two lepton family chemical potentials $\mu_{L_{e}}$ and $\mu_{L_{\mu}}$. After these are fixed, the values of the pressure in all competing neutral phases of quark matter should be compared. This is determined by using the same algorithm as in Ref. [5]. The complete set of equations (11) and (12) is solved for each of the eight phases allowed by symmetries. Then, the corresponding values of the pressure are determined from Eq. (10). The phase with the largest pressure is the ground state.

In the present paper, we always assume that the muon lepton-number chemical potential vanishes, i.e., $\mu_{L_{\mu}}=0$. This is expected to be a good approximation for matter inside a protoneutron star. Our analysis can thus be interpreted as an extension of the $T-\mu$ phase diagram discussed in Ref. [5] into the $\mu_{L_{e}}$ direction. Consequently, the complete phase structure requires a threedimensional presentation.

\section{A. Three-dimensional phase diagram}

The general features of the phase diagram in the threedimensional space spanned by the quark chemical potential $\mu$, the lepton-number chemical potential $\mu_{L_{e}}$, and the temperature $T$ are depicted in Fig. 22 Because of a rather complicated structure of the diagram, only the four main phases $(\chi \mathrm{SB}, \mathrm{NQ}, 2 \mathrm{SC}$ and $\mathrm{CFL})$ are shown explicitly. Although it is not labeled, a thin slice of a fifth phase, the uSC phase, squeezed in between the 2SC and CFL phases, can also be seen. While lacking detailed information, the phase diagram in Fig. 2 gives a clear overall picture. Among other things, one sees, for example, that the CFL phase becomes strongly disfavored with increasing $\mu_{L_{e}}$ and gets gradually replaced by the $2 \mathrm{SC}$ phase.

In order to discuss the structure of the phase diagram in more detail we proceed by showing several twodimensional slices. These are obtained by keeping one of the chemical potentials, $\mu$ or $\mu_{L_{e}}$, fixed and varying the other two parameters.

\section{B. $T-\mu$ phase diagram}

We begin with presenting the phase diagrams at three fixed values of the lepton-number chemical potential, $\mu_{L_{e}}=0$ (upper panel), $\mu_{L_{e}}=200 \mathrm{MeV}$ (middle panel) and $\mu_{L_{e}}=400 \mathrm{MeV}$ (lower panel), in Fig. 3 The first of them has already been discussed in Ref. [5]. Here we show it again only for the purpose of comparison with the other two diagrams. The general effect of neutrino trapping can be understood by analyzing the similarities and differences between the three diagrams. Note that, in this paper, we use the same convention for line styles as in Ref. [5]: thick and thin solid lines denote first- and second-order phase transitions, respectively; dashed lines indicate the (dis-)appearance of gapless modes in different phases.

Here it is appropriate to note that, in the same model, a schematic version of the $T-\mu$ phase diagram at $\mu_{L_{e}}=$ $200 \mathrm{MeV}$ was first presented in Ref. [8], see the right panel of Fig. 4 there. If one ignores the complications due to the presence of the uSC phase and various gapless phases, the results of Ref. [8] are in qualitative agreement with our findings here.

In order to understand the basic characteristics of different phases in the phase diagrams in Fig. 3 we also present the results for the dynamical quark masses, the gap parameters, and the charge chemical potentials. These are plotted as functions of the quark chemical potential in Figs. 4 and 5 for two different values of the temperature in the case of $\mu_{L_{e}}=200 \mathrm{MeV}$ and $\mu_{L_{e}}=400 \mathrm{MeV}$, respectively.

In each of the diagrams, there are roughly four distinct regimes. At low temperature and low quark chemical potential, there is a region in which the approximate chiral symmetry is spontaneously broken by large $\bar{\psi} \psi$-condensates. This phase is denoted by $\chi \mathrm{SB}$. In this regime, quarks have relatively large constituent masses which are close to the vacuum values, see Figs. 4 and [5 Here the density of all quark flavors is very low and even vanishes at $T=0$. There is no diquark pairing in this phase. The $\chi \mathrm{SB}$ phase is rather insensitive to the presence of a nonzero lepton-number chemical potential. With increasing $\mu_{L_{e}}$ the phase boundary is only slightly shifted to lower values of $\mu$. This is just another manifestation of the strengthening of the 2SC phase due to neutrino trapping.

With increasing temperature, the $\bar{\psi} \psi$-condensates melt and the $\chi \mathrm{SB}$ phase turns into the normal quark matter (NQ) phase where the quark masses are relatively small. Because of the explicit breaking of the chiral symmetry by the current quark masses, there is no need for a phase transition between the two regimes. In fact, at low chemical potentials we find only a smooth cross-over, whereas there is a first-order phase transition in a limited region, $320 \lesssim \mu \lesssim 360 \mathrm{MeV}$. In contrast to the $\chi \mathrm{SB}$ regime, the high-temperature NQ phase extends to arbitrary large values of $\mu$. All these qualitative features are little affected by the lepton-number chemical potential.

The third regime is located at relatively low temperatures but at quark chemical potentials higher than in the $\chi \mathrm{SB}$ phase. In this region, the masses of the up and down quarks have already dropped to values well below their respective chemical potentials while the strange quark mass is still large, see left columns of panels in Figs. 4 and 5 As a consequence, up and down quarks are quite 
abundant but strange quarks are essentially absent.

It turns out that the detailed phase structure in this region is very sensitive to the lepton-number chemical potential. At $\mu_{L_{e}}=0$, as discussed in Ref. [5], the pairing between up and down quarks is strongly hampered by the constraints of neutrality and $\beta$ equilibrium. As a consequence, there is no pairing at low temperatures, $T \lesssim 10 \mathrm{MeV}$, and g2SC-type pairing appears at moderate temperatures, $10 \mathrm{MeV} \lesssim T<T_{c}$ with the value of $T_{c}$ in the range of several dozen $\mathrm{MeV}$, see the upper panel in Fig. 3] The situation changes dramatically with increasing the value of the lepton-number chemical potential. Eventually, the low-temperature region of the normal phase of quark matter is replaced by the (g)2SC phase (e.g., at $\mu=400 \mathrm{MeV}$, this happens at $\mu_{L_{e}} \simeq 110 \mathrm{MeV}$ ). With $\mu_{L_{e}}$ increasing further, no qualitative changes happen in this part of the phase diagram, except that the area of the (g)2SC phase expands slightly.

Finally, the region in the phase diagram at low temperatures and large quark chemical potentials corresponds to phases in which the cross-flavor strange-nonstrange Cooper pairing becomes possible. In general, as the strength of pairing increases with the quark chemical potential, the system passes through regions of the gapless uSC (guSC), uSC, and gCFL phases and finally reaches the $\mathrm{CFL}$ phase. (Of course, the intermediate phases may not always be realized.) The effect of neutrino trapping, which grows with increasing the leptonnumber chemical potential, is to push out the location of the strange-nonstrange pairing region to larger values of $\mu$. Of course, this is in agreement with the general arguments in Sec. III

It is interesting to note that the growth of the strangeness content with increasing quark chemical potential could indirectly be deduced from the behavior of the electric charge chemical potential $\mu_{Q}$ at $T=0$, see the solid lines in the right panels in Figs. 4 and 5 The value of $\mu_{Q}$ reaches its minimum somewhere in a range of values of the quark chemical potential around $\mu \simeq 440 \mathrm{MeV}$. This corresponds to the point where the strange quark chemical potential $\mu_{s} \simeq \mu-\mu_{Q} / 3$ reaches the value of the strange quark mass (see left panels). Hence, there are essentially no strange quarks at lower values of $\mu$, and a rapidly increasing amount of strange quarks at higher values of $\mu$. Since the latter contribute to the electric neutralization, this is a natural explanation for the drop of $\left|\mu_{Q}\right|$ above this point.

As we mentioned earlier, the presence of the leptonnumber chemical potential $\mu_{L_{e}}$ leads to a change of the quark Fermi momenta. This change in turn affects Cooper pairing of quarks, facilitating the appearance of some phases and suppressing others. As it turns out, there is also another qualitative effect due to a nonzero value of $\mu_{L_{e}}$. In particular, we find several new variants of gapless phases which did not exist at vanishing $\mu_{L_{e}}$. In Figs. [3] and 7] these are denoted by the same names, g2SC or gCFL, but with one or two primes added.

We define the g2SC' as the gapless two-flavor color-
TABLE I: Definitions of gapless phases.

\begin{tabular}{lll}
\hline \hline Name & Gapless mode(s) & Diquark condensate(s) \\
& $\epsilon(k) \sim\left|k-k_{F}^{\mathrm{eff}}\right|$ & \\
\hline g2SC & $r u-g d, g u-r d$ & $\Delta_{3}$ \\
g2SC' $^{\prime}$ & $r d-g u, g d-r u$ & $\Delta_{3}$ \\
guSC & $r s-b u$ & $\Delta_{2}, \Delta_{3}$ \\
gCFL & $g s-b d$ & $\Delta_{1}, \Delta_{2}, \Delta_{3}$ \\
gCFL' $^{\prime}$ & $r s-b u$ & $\Delta_{1}, \Delta_{2}, \Delta_{3}$ \\
gCFL" $^{\prime \prime}$ & $g s-b d, r s-b u$ & $\Delta_{1}, \Delta_{2}, \Delta_{3}$ \\
\hline \hline
\end{tabular}

superconducting phase in which the gapless excitations correspond to $r d-g u$ and $g d-r u$ modes instead of the usual $r u-g d$ and $g u-r d$ ones, i.e., $u$ and $d$ flavors are exchanged as compared to the usual g2SC phase. The g2SC' phase becomes possible only when the value of $\left(\mu_{r u}-\mu_{g d}\right) / 2 \equiv\left(\mu_{Q}+\mu_{3}\right) / 2$ is positive and larger than $\Delta_{3}$. The other phases are defined in a similar manner. In particular, the $\mathrm{gCFL}^{\prime}$ phase, already introduced in Sec. III is indicated by the gapless $r s-b u$ mode, while the $\mathrm{gCFL}^{\prime \prime}$ phase has both $g s-b d$ (as in the gCFL phase) and $r s-b u$ gapless modes. Definitions of all gapless phases are summarized in Table 1

\section{Lepton fraction $Y_{L_{e}}$}

Our numerical results for the lepton fraction $Y_{L_{e}}$ are shown in Fig. 6] The thick and thin lines correspond to two different fixed values of the lepton-number chemical potential, $\mu_{L_{e}}=200 \mathrm{MeV}$ and $\mu_{L_{e}}=400 \mathrm{MeV}$, respectively. For a fixed value of $\mu_{L_{e}}$, we find that the lepton fraction changes only slightly with temperature. This is concluded from the comparison of the results at $T=0$ (solid lines), $T=20 \mathrm{MeV}$ (dashed lines), and $T=40 \mathrm{MeV}$ (dotted lines) in Fig. 6]

As is easy to check, at $T=40 \mathrm{MeV}$, i.e., when Cooper pairing is not so strong, the $\mu$ dependence of $Y_{L_{e}}$ does not differ very much from the prediction in the simple twoflavor model in Sec. III] By saying this, of course, one should not undermine the fact that the lepton fraction in Fig. 6] has a visible structure in the dependence on $\mu$ at $T=0$ and $T=20 \mathrm{MeV}$. This indicates that quark Cooper pairing plays a nontrivial role in determining the value of $Y_{L_{e}}$.

Our numerical study shows that it is hard to achieve values of the lepton fraction more than about 0.05 in the CFL phase. Gapless versions of the CFL phases, on the other hand, could accommodate a lepton fraction up to about 0.2 or so, provided the quark and lepton-number chemical potentials are sufficiently large.

From Fig.6] we can also see that the value of the lepton fraction $Y_{L_{e}} \approx 0.4$, i.e., the value expected at the center of the protoneutron star right after its creation, requires the lepton-number chemical potential $\mu_{L_{e}}$ in the range somewhere between $200 \mathrm{MeV}$ and $400 \mathrm{MeV}$, or slightly 
higher. The larger the quark chemical potential $\mu$ the larger $\mu_{L_{e}}$ is needed. Then, in a realistic construction of a star, this is likely to result in a noticeable gradient of the lepton-number chemical potential at the initial time. This gradient may play an important role in the subsequent deleptonization due to neutrino diffusion through dense matter.

\section{D. $T-\mu_{L_{e}}$ phase diagram}

Now let us explore the phase diagram in the plane of temperature and lepton-number chemical potential, keeping the quark chemical potential fixed. Two such slices of the phase diagram are presented in Fig. 7 The upper panel corresponds to a moderate value of the quark chemical potential, $\mu=400 \mathrm{MeV}$. This could be loosely termed as the "outer stellar core" phase diagram. The lower panel in Fig. 7 corresponds to $\mu=500 \mathrm{MeV}$, and we could associate it with the "inner stellar core" case. Note, however, that the terms "inner core" and "outer core" should not be interpreted literally here. The central densities of (proto-)neutron stars are subject to large theoretical uncertainties and, thus, are not known very well. In the model at hand, the case $\mu=400 \mathrm{MeV}$ ("outer core") corresponds to a range of densities around $4 \rho_{0}$, while the case $\mu=500 \mathrm{MeV}$ ("inner core") corresponds to a range of densities around $10 \rho_{0}$. These values are of the same order of magnitude that one typically obtains in models (see, e.g., Ref. [30]).

In addition to the phase diagrams, we also show the results for the dynamical quark masses, the gap parameters, and the charge chemical potentials. These are plotted as functions of temperature in Fig. $8(\mu=400 \mathrm{MeV}$, "outer core" case) and in Fig. 9] ( $\mu=500 \mathrm{MeV}$, "inner core" case), for three different values of the leptonnumber chemical potential, $\mu_{L_{e}}=0 \mathrm{MeV}$ (upper panels), $\mu_{L_{e}}=200 \mathrm{MeV}$ (middle panels) and $\mu_{L_{e}}=400 \mathrm{MeV}$ (lower panels).

At first sight, the two diagrams in Fig. 7 look so different that no obvious connection between them could be made. It is natural to ask, therefore, how such a dramatic change could happen with increasing the value of the quark chemical potential from $\mu=400 \mathrm{MeV}$ to $\mu=500 \mathrm{MeV}$. In order to understand this, it is useful to place the corresponding slices of the phase diagram in the three-dimensional diagram in Fig. 2

The $\mu=500 \mathrm{MeV}$ diagram corresponds to the righthand side surface of the bounding box in Fig. 2. This contains almost all complicated phases with strangenonstrange cross-flavor pairing. The $\mu=400 \mathrm{MeV}$ diagram, on the other hand, is obtained by cutting the three-dimensional diagram with a plane parallel to the bounding surface, but going through the middle of the diagram. This part of the diagram is dominated by the 2SC and NQ phases. Keeping in mind the general structure of the three-dimensional phase diagram, it is also not difficult to understand how the two diagrams in Fig. 0 transform into each other.

Several comments are in order regarding the zerotemperature phase transition from the CFL to gCFL' phase, shown by a small black triangle in the phase diagram at $\mu=500 \mathrm{MeV}$, see the lower panel in Fig. 7 The appearance of this transition is in agreement with the analytical result in Sec. III Moreover, the critical value of the lepton-number chemical potential also turns out to be very close to the estimate in Eq. (23). Indeed, by taking into account that $M_{s} \approx 214 \mathrm{MeV}$ and $\Delta_{2} \approx 76 \mathrm{MeV}$, we obtain $\mu_{L_{e}}^{(\mathrm{cr})}=\Delta_{2}-M_{s}^{2} /(2 \mu) \approx 30 \mathrm{MeV}$ which agrees well with the numerical value.

In order to estimate how the critical value of $\mu_{L_{e}}$ changes with decreasing the quark chemical potential below $\mu=500 \mathrm{MeV}$, we can use the zero-temperature numerical results for $M_{s}$ and $\Delta_{2}$ from Ref. [5]. Then, we arrive at the following power-law fit for the $\mu$-dependence of the critical value:

$$
\mu_{L_{e}}^{(\mathrm{cr})} \approx \frac{23}{4000}(\mu-457.4)(622.1-\mu),
$$

for $457.4 \leq \mu \leq 500 \mathrm{MeV}$ (in the fit, $\mu_{L_{e}}^{(\mathrm{cr})}$ and $\mu$ are measured in $\mathrm{MeV}$ ). Note that the CFL phase does not appear at $T=0$ when $\mu<457.4 \mathrm{MeV}[5]$. With increasing the values of the quark chemical potential above $\mu=500 \mathrm{MeV}$, one expects that the critical value of $\mu_{L_{e}}$ should continue to increase for a while, and then decrease when the effects of the cutoff start to suppress the size of the gap $\Delta_{2}$. However, the validity of the fit in Eq. (26) is questionable there because no numerical data for $\mu>500 \mathrm{MeV}$ was used in its derivation.

Before concluding this subsection, we should mention that a schematic version of the phase diagram in $T-\mu_{L_{e}}$ plane was earlier presented in Ref. [8], see the left panel in Fig. 4 there. In Ref. [8], the value of the quark chemical potential was $\mu=460 \mathrm{MeV}$, and therefore a direct comparison with our results is not simple. One can see, however, that the diagram of Ref. 8] fits naturally into the three-dimensional diagram in Fig. 2 Also, the diagram of Ref. 8] is topologically close to our version of the phase diagram at $\mu=500 \mathrm{MeV}$, shown in the lower panel of Fig. 7 The quantitative difference is not surprising: the region of the (g)CFL phase is considerably larger at $\mu=500 \mathrm{MeV}$ than at $\mu=460 \mathrm{MeV}$.

\section{CONCLUSIONS}

In this paper, we studied the effect of neutrino trapping on the phase diagram of neutral three-flavor quark matter within the NJL model of Ref. [25]. The results are obtained in the mean-field approximation, treating constituent quark masses as dynamically generated quantities. The overall structure of the phase diagram in the space of three parameters, namely temperature $T$, quark chemical potential $\mu$, and lepton-number chemical potential $\mu_{L_{e}}$, is summarized in Fig. 2. This is further detailed 
in several two-dimensional slices of the phase diagram, including diagrams in the plane of temperature and quark chemical potential (see Fig. 31) and in the plane of temperature and lepton-number chemical potential (see Fig. [7).

By making use of simple model-independent arguments, as well as detailed numerical calculations in the framework of an NJL-type model, we find that neutrino trapping helps Cooper pairing in the 2SC phase and suppresses the CFL phase. In essence, this is the consequence of satisfying the electric neutrality constraint in the quark system. In two-flavor quark matter, the (positive) leptonnumber chemical potential $\mu_{L_{e}}$ helps to provide extra electrons without inducing a large mismatch between the Fermi momenta of up and down quarks. With reducing the mismatch, of course, Cooper pairing gets stronger. This is in sharp contrast to the situation in the CFL phase of quark matter, which is neutral in the absence of electrons. Additional electrons due to large $\mu_{L_{e}}$ can only put extra stress on the system.

In application to protoneutron stars, our findings in this paper strongly suggest that the CFL phase is very unlikely to appear during the early stage of the stellar evolution before the deleptonization is completed. If color superconductivity occurs there, the 2SC phase is the best candidate for the ground state. In view of this, it might be quite natural to suggest that matter inside protoneutron stars contains little or no strangeness (just as the cores of the progenitor stars) during the early times of their evolution. In this connection, it is appropriate to recall that neutrino trapping also suppresses the appearance of strangeness in the form of hyperonic matter and kaon condensation [30]. Our results, therefore, are a special case of a generic property.

After the deleptonization occurs, it is possible that the ground state of matter at high density in the central region of the star is the CFL phase. This phase contains a large number of strange quarks. Therefore, an abundant production of strangeness should happen right after the deleptonization in the protoneutron star. If realized in nature, in principle this scenario may have observational signatures.

\section{Acknowledgments}

This work was supported in part by the Virtual Institute of the Helmholtz Association under grant No. VH-VI-041 and by Gesellschaft für Schwerionenforschung (GSI) and by Bundesministerium für Bildung und Forschung (BMBF). S. R. thanks for using the Center for Scientific Computing (CSC) of the Johann Wolfgang Goethe-Universität Frankfurt am Main.
[1] S. B. Rüster, I. A. Shovkovy, and D. H. Rischke, Nucl. Phys. A 743, 127 (2004).

[2] K. Fukushima, C. Kouvaris, and K. Rajagopal, Phys. Rev. D 71, 034002 (2005).

[3] I. A. Shovkovy, S. B. Rüster, and D. H. Rischke, J. Phys. G 31, S849 (2005).

[4] H. Abuki, M. Kitazawa, and T. Kunihiro, Phys. Lett. B 615, 102 (2005).

[5] S. B. Rüster, V. Werth, M. Buballa, I. A. Shovkovy, and D. H. Rischke, Phys. Rev. D 72, 034004 (2005).

[6] D. Blaschke, S. Fredriksson, H. Grigorian, A. M. Öztaş, and F. Sandin, Phys. Rev. D 72, 065020 (2005).

[7] M. Alford and K. Rajagopal, JHEP 0206, 031 (2002).

[8] A. W. Steiner, S. Reddy, and M. Prakash, Phys. Rev. D 66, 094007 (2002).

[9] M. Huang, P. F. Zhuang, and W. Q. Chao, Phys. Rev. D 67, 065015 (2003).

[10] I. Shovkovy and M. Huang, Phys. Lett. B 564, 205 (2003); M. Huang and I. Shovkovy, Nucl. Phys. A 729, 835 (2003).

[11] M. Alford, C. Kouvaris, and K. Rajagopal, Phys. Rev. Lett. 92, 222001 (2004); Phys. Rev. D 71, 054009 (2005).

[12] K. Iida, T. Matsuura, M. Tachibana, and T. Hatsuda, Phys. Rev. Lett. 93, 132001 (2004).

[13] K. Rajagopal and F. Wilczek, hep-ph/0011333 M. Alford, Ann. Rev. Nucl. Part. Sci. 51, 131 (2001); T. Schäfer, hep-ph/0304281 D. H. Rischke, Prog. Part. Nucl. Phys. 52, 197 (2004); M. Buballa, Phys. Rep. 407, 205 (2005); H.-C. Ren, hep-ph/0404074 M. Huang, Int. J. Mod. Phys. E 14, 675 (2005); I. A. Shovkovy, Found.
Phys. 35, 1309 (2005).

[14] M. G. Alford, K. Rajagopal, and F. Wilczek, Nucl. Phys. B537, 443 (1999).

[15] I. A. Shovkovy and L. C. R. Wijewardhana, Phys. Lett. B 470, 189 (1999); T. Schäfer, Nucl. Phys. B575, 269 (2000).

[16] M. Alford, K. Rajagopal, and F. Wilczek, Phys. Lett. B 422, 247 (1998); R. Rapp, T. Schäfer, E. V. Shuryak, and M. Velkovsky, Phys. Rev. Lett. 81, 53 (1998).

[17] D. T. Son, Phys. Rev. D 59, 094019 (1999); T. Schäfer and F. Wilczek, Phys. Rev. D 60, 114033 (1999); D. K. Hong, V. A. Miransky, I. A. Shovkovy, and L. C. R. Wijewardhana, Phys. Rev. D 61, 056001 (2000); R. D. Pisarski and D. H. Rischke, Phys. Rev. D 61, 051501(R) (2000); W. E. Brown, J. T. Liu, and H.C. Ren, Phys. Rev. D 61, 114012 (2000).

[18] F. Neumann, M. Buballa, and M. Oertel, Nucl. Phys. A 714, 481 (2003); I. Shovkovy, M. Hanauske, and M. Huang, Phys. Rev. D 67, 103004 (2003); S. Reddy and G. Rupak, Phys. Rev. C 71, 025201 (2005).

[19] T. Schäfer, Phys. Rev. D 62, 094007 (2000); M. Buballa, J. Hošek, and M. Oertel, Phys. Rev. Lett. 90, 182002 (2003); A. Schmitt, Q. Wang, and D. H. Rischke, Phys. Rev. D 66, 114010 (2002); Phys. Rev. Lett. 91, 242301 (2003); A. Schmitt, Phys. Rev. D 71, 054016 (2005); D. N. Aguilera, D. Blaschke, M. Buballa, and V. L. Yudichev, Phys. Rev. D 72, 034008 (2005).

[20] M. G. Alford, J. A. Bowers, and K. Rajagopal, Phys. Rev. D 63, 074016 (2001); R. Casalbuoni, R. Gatto, M. Mannarelli, and G. Nardulli, Phys. Rev. D 66, 014006 
(2002); I. Giannakis, J. T. Liu, and H.-C. Ren, Phys. Rev. D 66, 031501(R) (2002).

[21] P. F. Bedaque and T. Schäfer, Nucl. Phys. A 697, 802 (2002); D. B. Kaplan and S. Reddy, Phys. Rev. D 65, 054042 (2002); A. Kryjevski, D. B. Kaplan, and T. Schäfer, Phys. Rev. D 71, 034004 (2005); A. Kryjevski, hep-ph/0508180 T. Schäfer, hep-ph/0508190

[22] D. K. Hong, hep-ph/0506097

[23] E. V. Gorbar, M. Hashimoto, and V. A. Miransky, Phys. Lett. B 632, 305 (2006).

[24] M. Huang and I. A. Shovkovy, Phys. Rev. D 70, 051501(R) (2004); Phys. Rev. D 70, 094030 (2004); R. Casalbuoni, R. Gatto, M. Mannarelli, G. Nardulli, and M. Ruggieri, Phys. Lett. B 605, 362 (2005); I. Giannakis and H.-C. Ren, Phys. Lett. B 611, 137 (2005); M. Alford and Q. H. Wang, J. Phys. G 31, 719 (2005).
[25] P. Rehberg, S. P. Klevansky, and J. Hüfner, Phys. Rev. C 53, 410 (1996).

[26] M. Buballa and M. Oertel, Nucl. Phys. A 703, 770 (2002).

[27] M. Huang, P. F. Zhuang, and W. Q. Chao, Phys. Rev. D 65, 076012 (2002).

[28] K. Rajagopal and F. Wilczek, Phys. Rev. Lett. 86, 3492 (2001).

[29] K. Fukushima, hep-ph/0512138

[30] M. Prakash, I. Bombaci, M. Prakash, P. J. Ellis, J. M. Lattimer, and R. Knorren, Phys. Rept. 280, 1 (1997).

[31] M. Buballa and I. A. Shovkovy, Phys. Rev. D 72, 097501 (2005). 


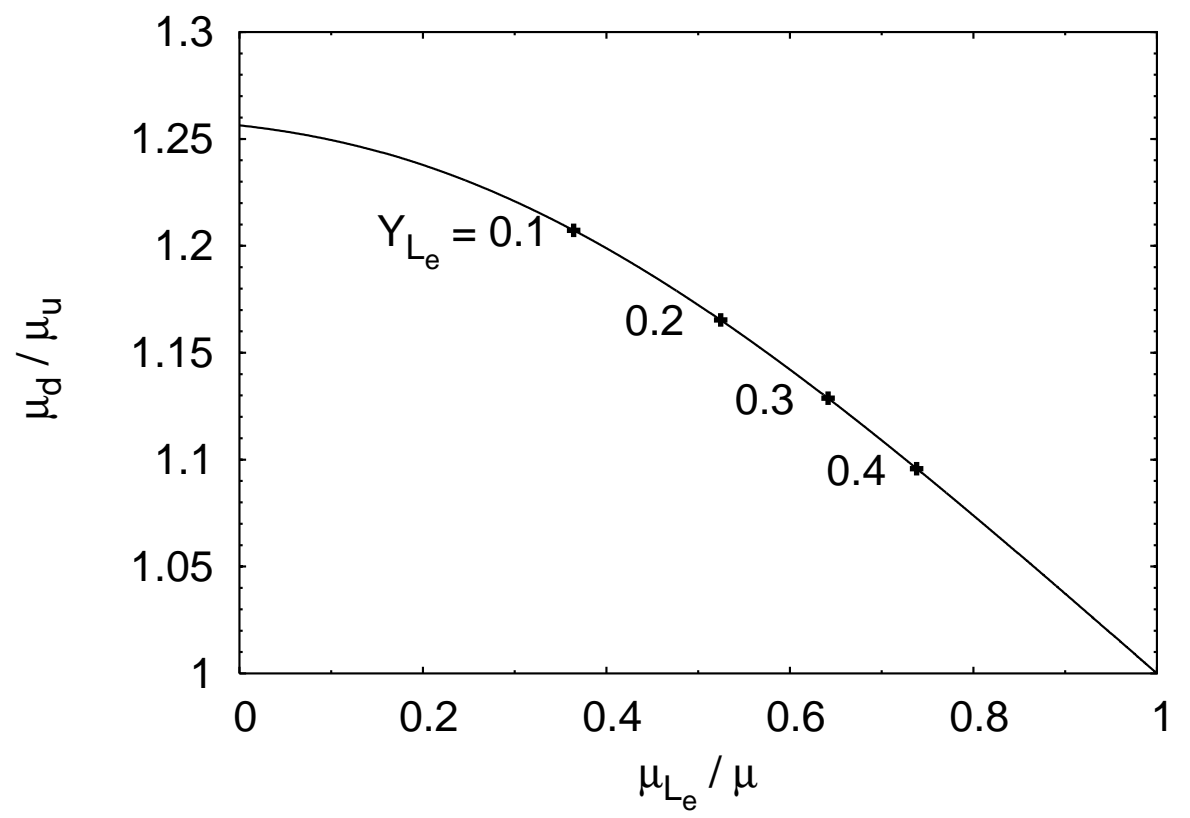

FIG. 1: Ratio of down and up quark chemical potentials as a function of $\mu_{L_{e}} / \mu$ in the toy model of Sec. III The crosses mark the solutions at several values of the lepton fraction.

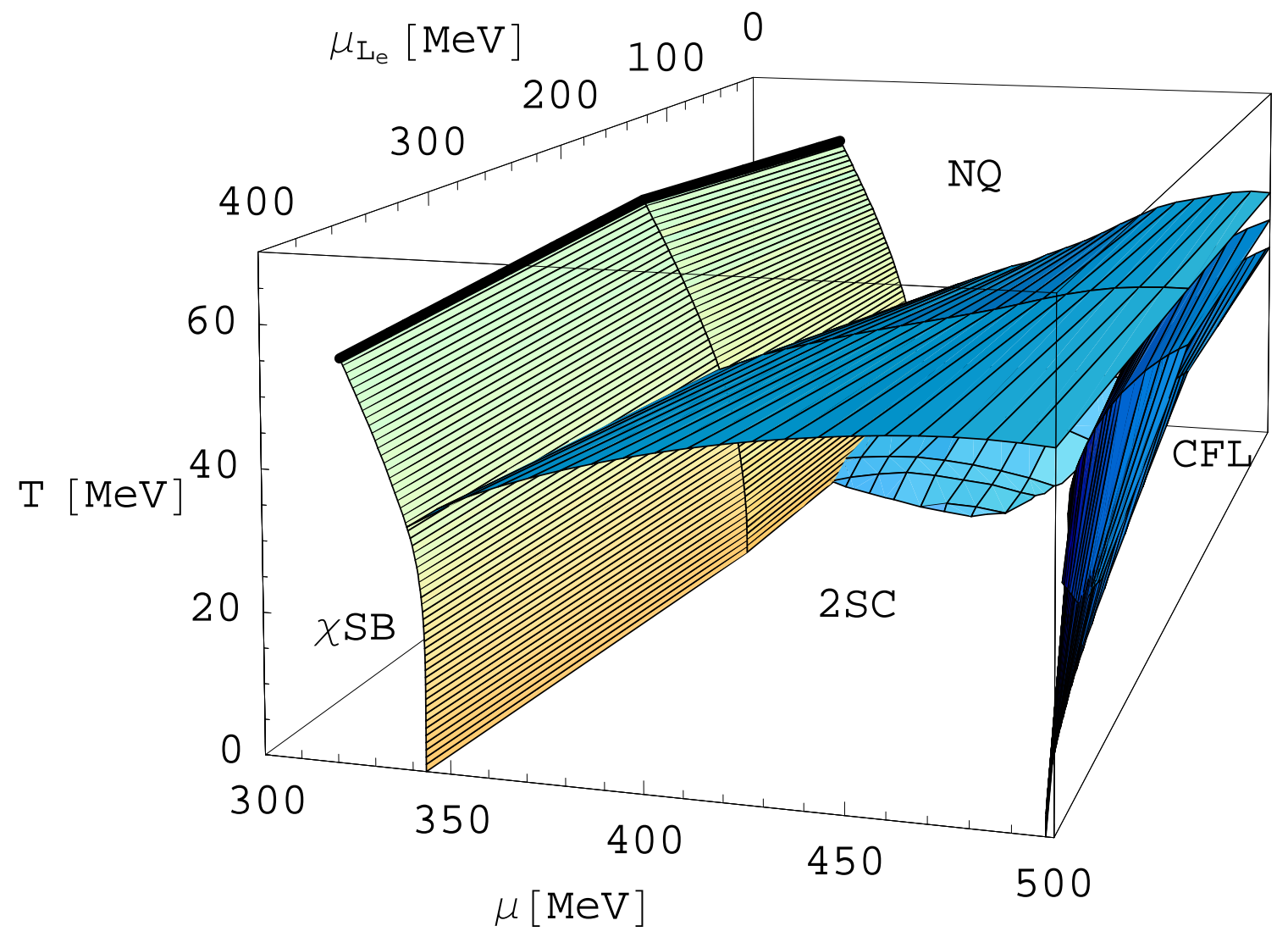

FIG. 2: General structure of the phase diagram of neutral dense quark matter in the three-dimensional space spanned by the quark chemical potential $\mu$, the lepton-number chemical potential $\mu_{L_{e}}$, and the temperature $T$. 

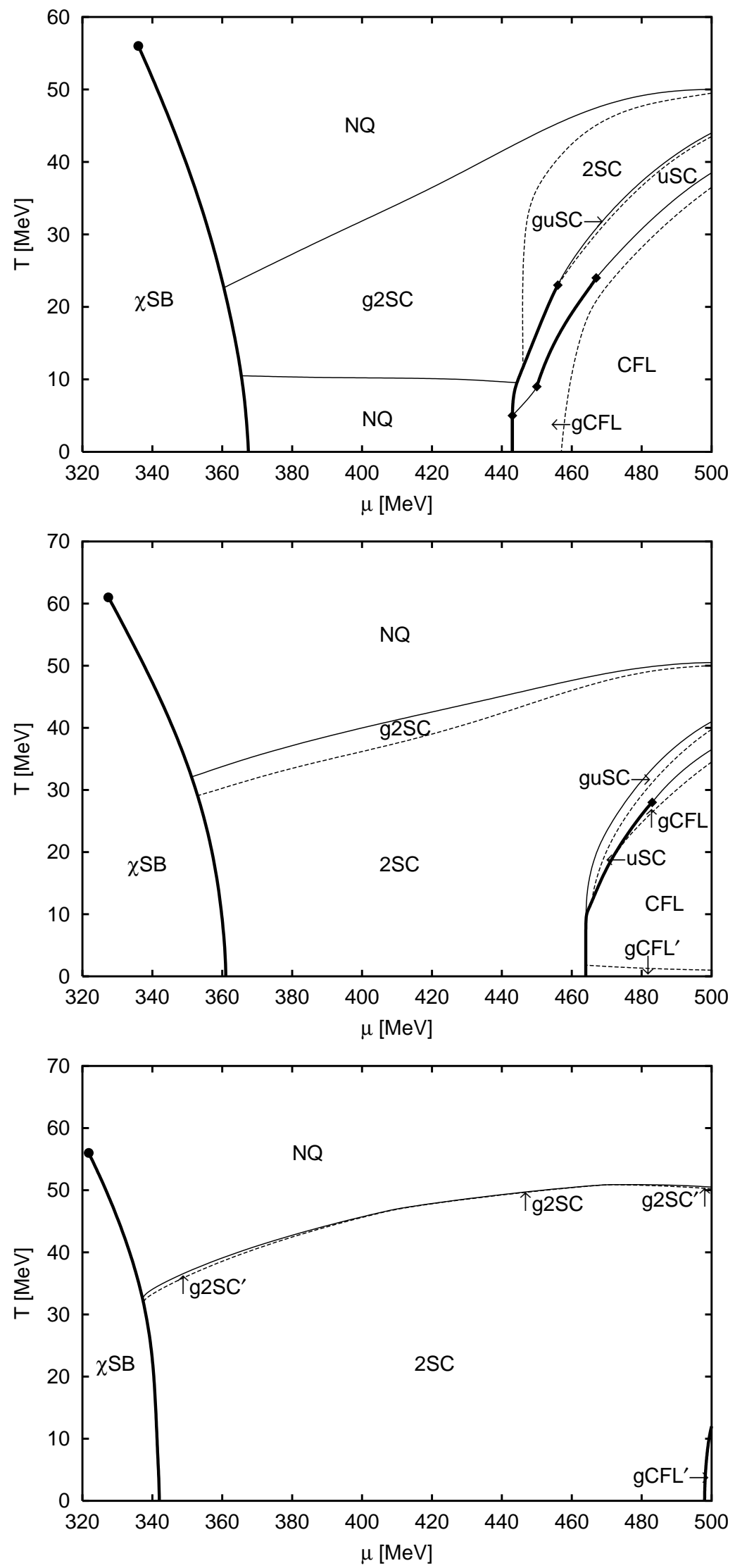

FIG. 3: The phase diagrams of neutral quark matter at fixed lepton-number chemical potentials $\mu_{L_{e}}=0$ (upper panel), $\mu_{L_{e}}=200 \mathrm{MeV}$ (middle panel), and $\mu_{L_{e}}=400 \mathrm{MeV}$ (lower panel). Note that the upper diagram, describing matter in absence of neutrino trapping, is the same as in Fig. 1 of Ref. [5]. 

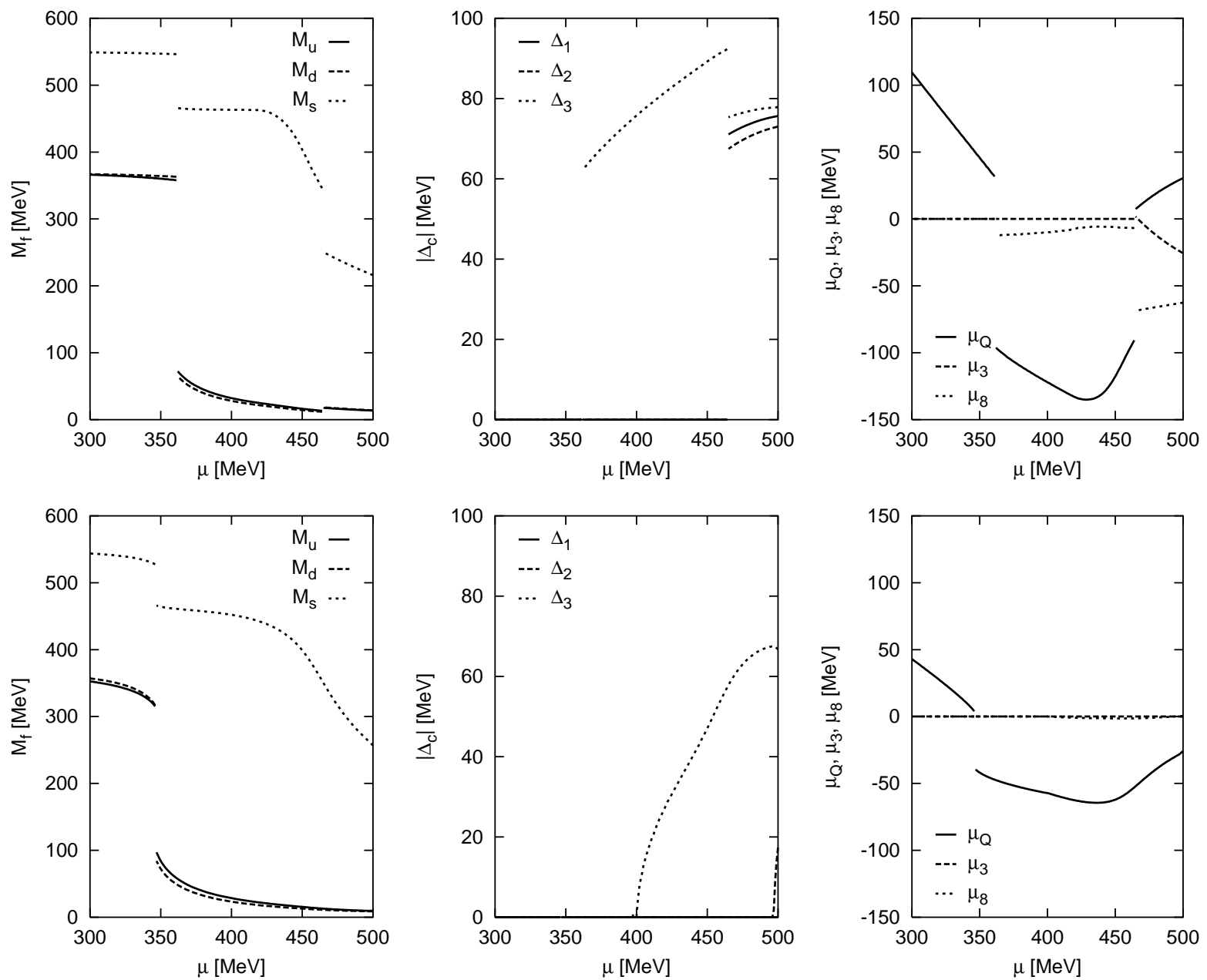

FIG. 4: Dependence of the quark masses, of the gap parameters, and of the electric and color charge chemical potentials on the quark chemical potential at a fixed temperature, $T=0 \mathrm{MeV}$ (three upper panels) and $T=40 \mathrm{MeV}$ (three lower panels). The lepton-number chemical potential is kept fixed at $\mu_{L_{e}}=200 \mathrm{MeV}$. 

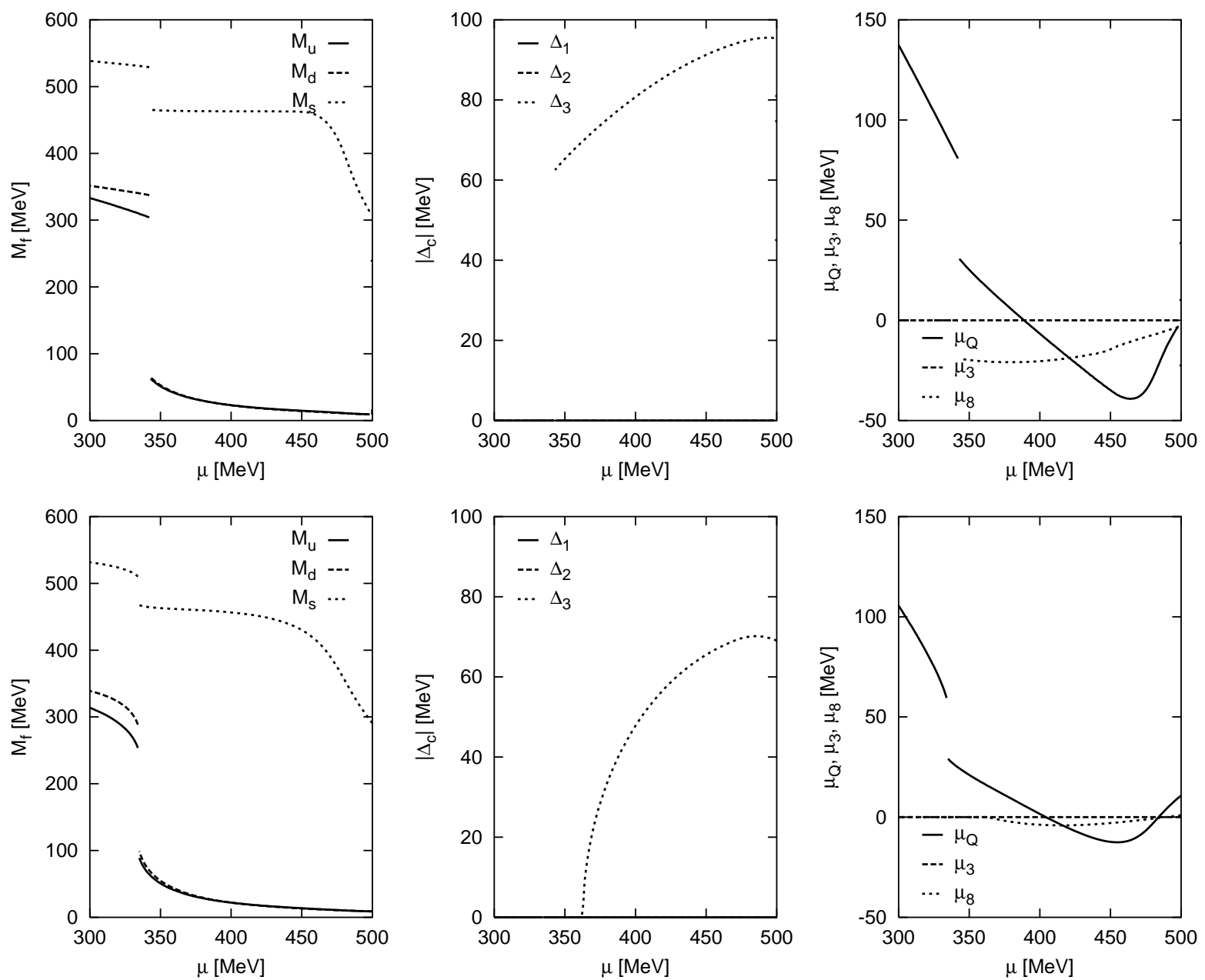

FIG. 5: Dependence of the quark masses, of the gap parameters, and of the electric and color charge chemical potentials on the quark chemical potential at a fixed temperature, $T=0 \mathrm{MeV}$ (three upper panels), and $T=40 \mathrm{MeV}$ (three lower panels). The lepton-number chemical potential is kept fixed at $\mu_{L_{e}}=400 \mathrm{MeV}$.

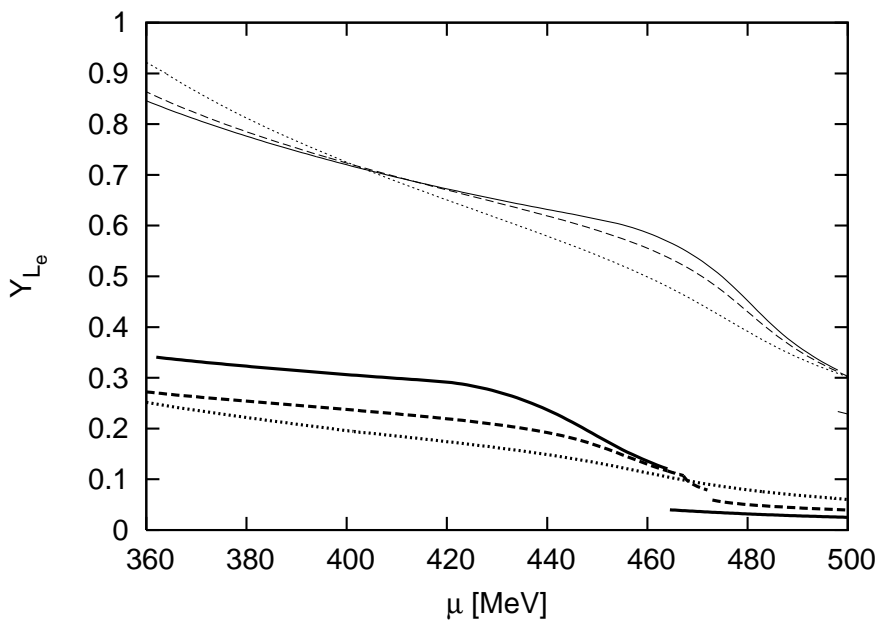

FIG. 6: Dependence of the electron family lepton fraction $Y_{L_{e}}$ for $\mu_{L_{e}}=200 \mathrm{MeV}$ (thick lines) and $\mu_{L_{e}}=400 \mathrm{MeV}$ (thin lines) on the quark chemical potential at a fixed temperature, $T=0 \mathrm{MeV}$ (solid lines), $T=20 \mathrm{MeV}$ (dashed lines), and $T=40 \mathrm{MeV}$ (dotted lines). 

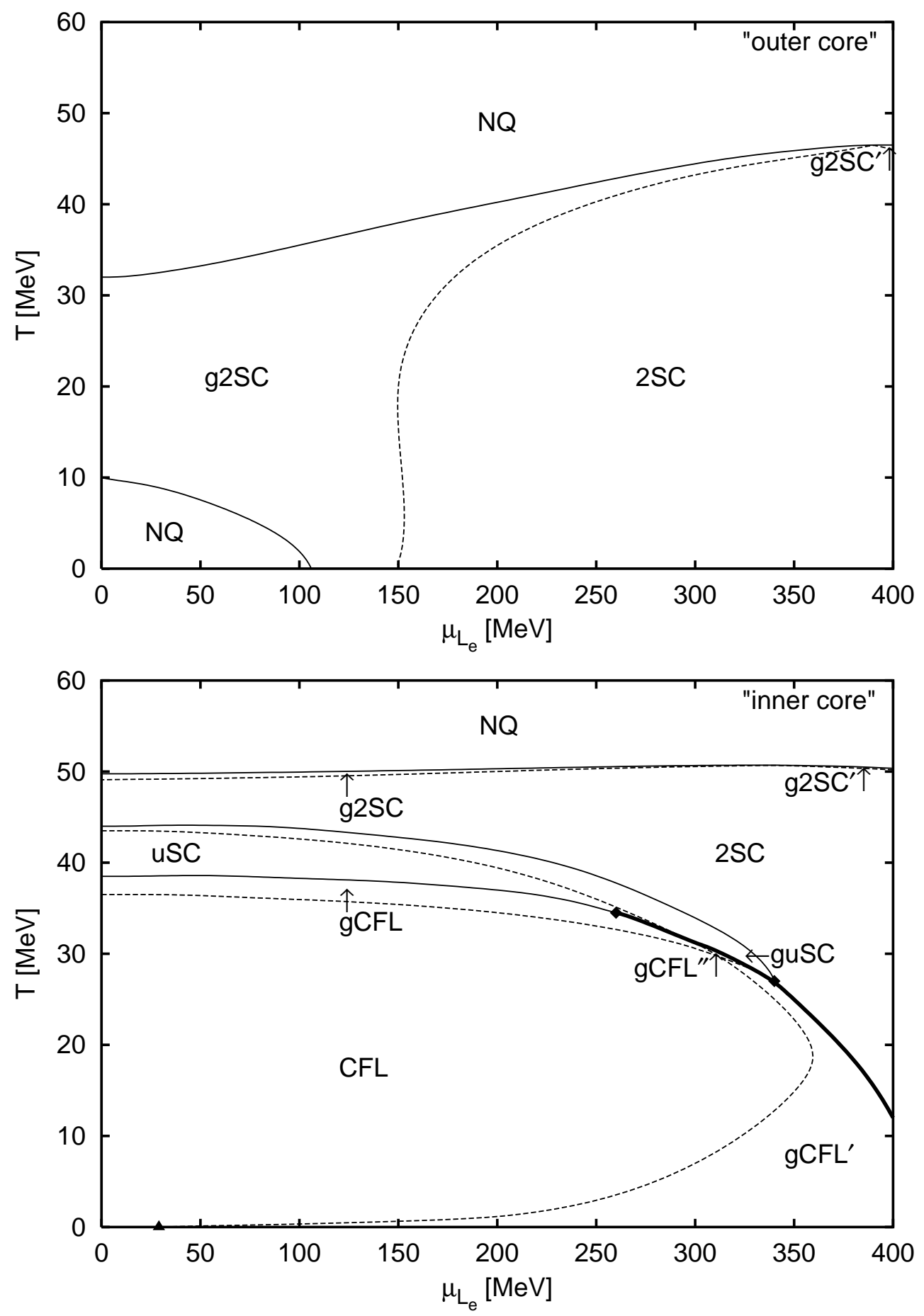

FIG. 7: The phase diagrams of neutral quark matter in the plane of temperature and lepton-number chemical potential at two fixed values of quark chemical potential: $\mu=400 \mathrm{MeV}$ (upper panel) and $\mu=500 \mathrm{MeV}$ (lower panel). The triangle in the lower panel denotes the transition point from the CFL phase to the gCFL' phase at $T=0$. 

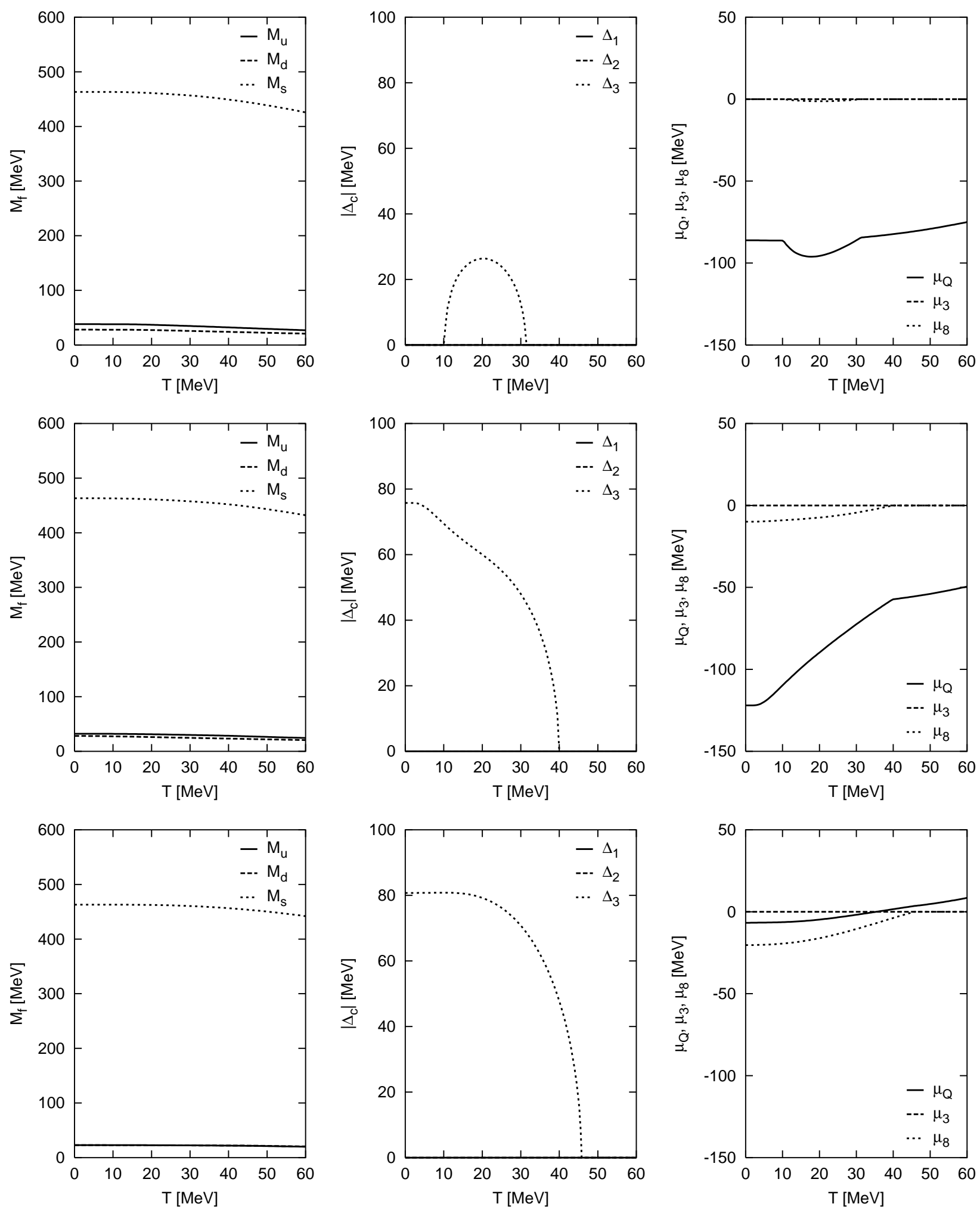

FIG. 8: Dependence of the quark masses, of the gap parameters, and of the electric and color charge chemical potentials on the temperature at a fixed value of the lepton-number chemical potential, $\mu_{L_{e}}=0 \mathrm{MeV}$ (three upper panels), $\mu_{L_{e}}=200 \mathrm{MeV}$ (three middle panels), and $\mu_{L_{e}}=400 \mathrm{MeV}$ (three lower panels). The quark chemical potential is $\mu=400 \mathrm{MeV}$ which may correspond to the conditions in the outer quark core inside a neutron star. 

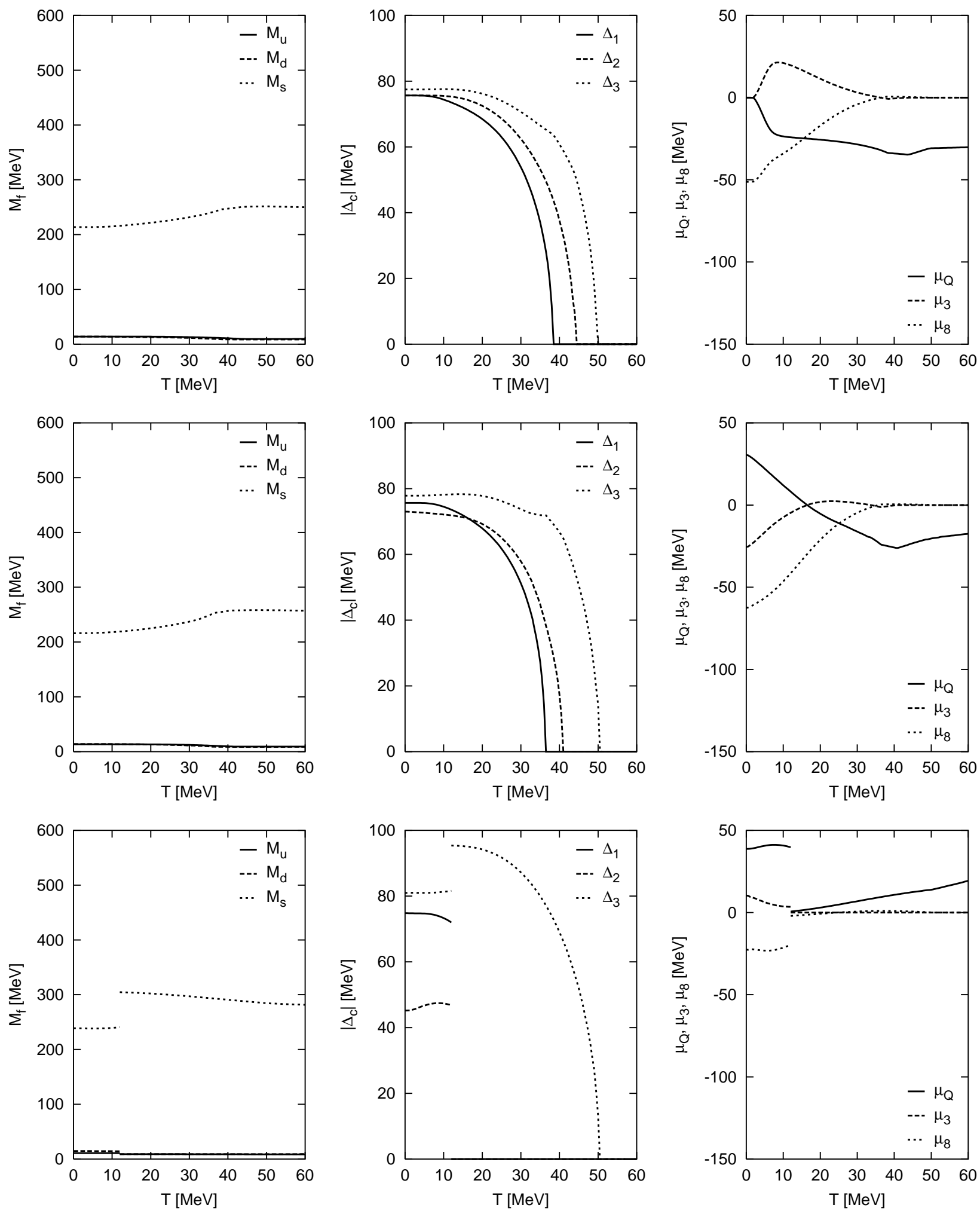

FIG. 9: Dependence of the quark masses, of the gap parameters, and of the electric and color charge chemical potentials on the temperature at a fixed value of the lepton-number chemical potential, $\mu_{L_{e}}=0 \mathrm{MeV}$ (three upper panels), $\mu_{L_{e}}=200 \mathrm{MeV}$ (three middle panels), and $\mu_{L_{e}}=400 \mathrm{MeV}$ (three lower panels). The quark chemical potential is $\mu=500 \mathrm{MeV}$ which may correspond to the conditions at the center of a quark core inside a neutron star. 